\title{
Characterization of the highly immunogenic VP2 protrusion domain as a diagnostic antigen for members of Birnaviridae family
}

\author{
Maryam Zafar ${ }^{1,2,3} \cdot$ Majid Ali Shah ${ }^{1,2} \cdot$ Aamir Shehzad $^{1,2} \cdot$ Anam Tariq $^{1,2} \cdot$ Mudasser Habib $^{4}$. \\ Muhammad Muddassar ${ }^{5}$ - Muhammad Salahuddin Shah ${ }^{4} \cdot$ Mazhar Iqbal $^{1,2} \cdot$ Farhid Hemmatzadeh $^{3}$ (D) \\ Moazur Rahman ${ }^{1,2,3}$ (D)
}

Received: 2 November 2019/Revised: 31 January 2020 / Accepted: 10 February 2020 / Published online: 23 February 2020

(C) Springer-Verlag GmbH Germany, part of Springer Nature 2020

\begin{abstract}
Birnaviridae is a family of viruses (birnaviruses) which consists of four genera, members of which cause diseases in fish, birds, mollusks, and insects. The genome of birnaviruses encodes the highly immunogenic VP2 capsid protein. In order to demonstrate that the VP2 protein can be exploited as a diagnostic antigen for birnaviruses, we developed a lateral flow assay based on the surface-exposed VP2 protrusion domain of a representative birnavirus, infectious bursal disease virus (IBDV) of serotype 1 which causes the highly devastating infectious bursal disease in chickens. The biophysical characterization of the purified domain reveals that the domain predominantly consists of $\beta$-sheets, exists in a trimeric form, and remains folded at high temperatures, making it suitable for diagnostic purposes. Owing to its highly immunogenic nature and excellent biophysical properties, we employed the VP2 protrusion domain in a gold nanoparticle-based lateral flow assay for rapid detection of anti-IBDV antibodies in serum samples of infected chickens. Our results indicate that the domain binds anti-IBDV antibodies with high specificity during laboratory testing and on-site testing. The lateral flow assay reported here yields comparable results in a qualitative manner as obtained through a commercial enzyme-linked immunosorbent assay (ELISA). As VP2 is a common capsid protein of birnaviruses, the lateral flow assay can be generalized for other birnaviruses, and members of Tetraviridae and Nodaviridae families which contain homologous VP2 capsid proteins.
\end{abstract}

Keywords Birnavirus $\cdot$ Circular dichroism $\cdot$ Gold nanoparticle-based lateral flow assay $\cdot$ Infectious bursal disease virus $\cdot$ Infrared spectroscopy $\cdot$ VP2 protrusion domain

Electronic supplementary material The online version of this article (https://doi.org/10.1007/s00253-020-10458-6) contains supplementary material, which is available to authorized users.

Farhid Hemmatzadeh

farhid.hemmatzadeh@adelaide.edu.au

Moazur Rahman

moazur.rahman@fulbrightmail.org; moaz@nibge.org

1 Drug Discovery and Structural Biology Group, Health Biotechnology Division, National Institute for Biotechnology and Genetic Engineering (NIBGE), Faisalabad, Pakistan

2 Pakistan Institute of Engineering and Applied Sciences (PIEAS), P.O. Nilore, Islamabad, Pakistan

3 School of Animal and Veterinary Sciences, Roseworthy Campus, The University of Adelaide, Roseworthy, South Australia, 5371, Australia

4 Vaccine Development Group, Animal Sciences Division, NIAB, Faisalabad, Pakistan

5 Department of Biosciences, COMSATS-University Islamabad, Park Road, Islamabad, Pakistan

\section{Introduction}

Birnaviridae is a family of viruses, commonly known as birnaviruses, which consists of four genera (Aquabirnavirus, Avibirnavirus, Blosnavirus, and Entomobirnavirus), members of which infect fish, birds, mollusks, and insects (Delmas et al. 2012). Two genera, Aquabirnavirus and Avibirnavirus, cause economically important diseases such as infectious pancreatic necrosis (IPN) in fish and infectious bursal disease (IBD) in birds, respectively (Hill and Way 1995; Vanden et al. 2000). The viral capsid of birnaviruses is mainly composed of VP2 protein which possesses enormous antigenic propensity (Azad et al. 1987; Böttcher et al. 1997; Pous et al. 2005; Letzel et al. 2007; Delmas et al. 2012; Taghavian et al. 2013; Wang et al. 2019). The highly immunogenic nature of the VP2 protein renders it an attractive target for use as a diagnostic antigen in immunoassays. 
Recently, lateral flow assays, also known as strip-based immunoassays, which are based on capsid proteins have gained considerable attention for detection of various pathogens since these assays are rapid, user-friendly, and costeffective (Sun et al. 2014; Sastre et al. 2016). Moreover, technical expertise or special instruments are not required for performing lateral flow assays. To the best of our knowledge, a lateral flow assay based on the VP2 capsid protein of birnaviruses has not been reported yet. As birnaviruses cause economically important diseases in fish and birds leading to enormous losses in fisheries and poultry industries worldwide, there is an urgent need to develop a lateral flow assay for timely detection of birnaviruses in infected animals. In order to demonstrate that the VP2 capsid protein can be used as a diagnostic antigen for detection of infections caused by birnaviruses, the surface-exposed VP2 protrusion domain of infectious bursal disease virus (IBDV), a representative member of Birnaviridae family, was selected for the development of a gold nanoparticle-based lateral flow assay. The surfaceexposed nature of the VP2 protrusion domain also renders it an attractive candidate for vaccine development.

The VP2 protrusion domain of IBDV is a part of the hypervariable region (Tyr206-Thr350) which plays an important role in the induction of neutralizing antibodies against the virus (Azad et al. 1987; Bayliss et al. 1990; Vakharia et al. 1994; Letzel et al. 2007). Moreover, the VP2 protrusion domain is exposed on the surface of the VP2 capsid protein, due to which it is highly immunogenic (Lee et al. 2006). Antigenic variations in the hypervariable region of IBDV lead to the emergence of pathogenic strains (such as very virulent (vvIBDV)) that can evade the host immune response (Vanden et al. 1996). In the late 1980s, vvIBDV (also known as hyper virulent strain (hvIBDV)) was first reported in Europe (Heine et al. 1991), which is responsible for causing severe (up to 100\%) mortality in immature chickens (Chettle et al. 1989). Importantly, strains of vvIBDV have rapidly circulated worldwide, being enzootic in Europe, America, Asia, and Africa (Jackwood and Nielsen 1997; Zorman et al. 2003; Banda et al. 2003; Stoute et al. 2013; Mohamed et al. 2014; Khan et al. 2017). IBD mainly affects the bursa of Fabricius with substantial destruction of B lymphocytes (Käufer and Weiss 1980). Acute infection with IBDV leads to various clinical signs including diarrhea, depression, ruffled feathers, anorexia, exhaustion, and in severe cases death of chickens (Ley et al. 1983). Several IBD outbreaks, caused by different strains of the virus, have been reported in various geographical regions of the world since the emergence of the disease in 1962 (Cosgrove 1962).

We present here the results obtained from the biophysical characterization of the purified VP2 protrusion domain in solution through spectroscopic techniques (mainly circular dichroism (CD) and Fourier-transform infrared (FTIR) spectroscopy) (Whitmore and Wallace 2004, 2008;
Goormaghtigh et al. 1994a, 1994b). For this purpose, the recombinant VP2 protrusion domain was heterologously expressed in the Escherichia coli expression system, and we applied biophysical methods to evaluate the stability and proper folding of the heterologously expressed recombinant protein before the development of the lateral flow device (LFD) for on-site detection of anti-IBDV antibodies in infected chickens. The data presented here indicate that the VP2 protrusion domain remains properly folded at elevated temperatures, suggesting that the domain can be used for practical purposes. Hence, the VP2 protrusion domain was exploited as a diagnostic antigen in a colloidal gold nanoparticle-based lateral flow assay for rapid detection of anti-IBDV antibodies. The assay shows promising results for the detection of anti-IBDV antibodies in serum samples and monitoring of immune responses and can be generalized for the detection of antibirnaviral antibodies in serum samples of other animals infected with birnaviruses.

\section{Materials and methods}

\section{Isolation of IBDV, extraction of viral RNA, and CDNA synthesis}

Viral particles of IBDV, a member of Birnaviridae family, were isolated from bursa of Fabricius of infected chickens. The detailed procedures for the isolation of virus, the extraction of viral RNA, and cDNA synthesis can be found in the Electronic Supplementary Material (ESM).

\section{Generation of expression construct}

The VP2 protrusion gene was introduced into the expression vector, pET28a-PreScission, following the protocol described previously (Shah et al. 2017) (see ESM for further details).

\section{Expression and purification of the VP2 protrusion domain}

For protein expression in E. coli cells, the expression vector (pET28a-PreS-VP2 protrusion domain) was transformed into the $E$. coli strain BL21 (DE3) (Novagen) and incubated overnight at $37^{\circ} \mathrm{C}$. A bunch of morphologically similar colonies, resistant to kanamycin $(30 \mu \mathrm{g} /$ $\mathrm{mL}$ ), were inoculated into $5-10 \mathrm{~mL}$ of fresh $\mathrm{LB}_{\text {kan }}$ broth (kanamycin $30 \mu \mathrm{g} / \mathrm{mL}$ ) and incubated for $16 \mathrm{~h}$ at $225 \mathrm{rpm}$ at $37{ }^{\circ} \mathrm{C}$. The culture was diluted 100 times in $\mathrm{LB}_{\text {kan }}$ broth and incubated at $37{ }^{\circ} \mathrm{C}$ with continuous shaking at $225 \mathrm{rpm}$ until the $\mathrm{OD}_{600}$ of the culture reached to 0.5-0.6. The expression of VP2 protrusion domain was induced by adding isopropyl- $\beta$-D-thiogalactopyranoside (IPTG) to a 
final concentration of $1 \mathrm{mM}$, and the cells were further grown for $5 \mathrm{~h}$ at $37^{\circ} \mathrm{C}$ and $220 \mathrm{rpm}$. Cells were harvested from the medium by centrifugation $(4000 \times \mathrm{g}$ for $30 \mathrm{~min})$ and resuspended in $50 \mathrm{~mL}$ lysis buffer $(20 \mathrm{mM}$ Tris-Cl, $\mathrm{pH} 7.9,300 \mathrm{mM} \mathrm{NaCl}$, and $4 \mathrm{mM} \beta$-mercaptoethanol (ME)). Following lysis of the cells at $25 \mathrm{Kpsi}$ using a cell disrupter (Constant System Ltd., UK), the supernatant was separated from the cell debris by centrifugation $(10,000 \times g$ for $30 \mathrm{~min}$ at $4{ }^{\circ} \mathrm{C}$ ) using a Beckman centrifuge. The supernatant was used to purify the soluble form of the VP2 protrusion domain through a nickel-nitrilotriacetate (Ni-NTA) agarose column (see ESM for further details). The eluted protein was dialyzed against buffer $(20 \mathrm{mM}$ Tris- $\mathrm{HCl}$ (pH 7.9), $100 \mathrm{mM} \mathrm{NaCl}$ ) at $4{ }^{\circ} \mathrm{C}$ overnight, and then concentrated to $5 \mathrm{mg} / \mathrm{mL}$ using an ultracentrifugal filter with a $10 \mathrm{kDa}$ molecular weight cut-off (Pierce, $88513)$. Gel filtration of the concentrated protein was performed at $4{ }^{\circ} \mathrm{C}$ using a Superdex 75 16/60 GL column connected to an ÄKTA system (GE Healthcare Life Sciences). The column was equilibrated with pre-chilled, degassed gel filtration buffer (20 mM Tris- $\mathrm{HCl}(\mathrm{pH} 7.9)$, $100 \mathrm{mM} \mathrm{NaCl}$ ). The purified protein was quantified using a NanoDrop 2000c spectrophotometer (Thermo Scientific), and the purity of the purified protein was analyzed by SDS-PAGE. The identity of the full-length VP2 protrusion domain was confirmed by mass spectrometry at Taplin MS Facility, Harvard Medical School, Boston (http://taplin.med.harvard.edu/), by peptide mass finger printing using chymotrypsin that cleaves at C-terminal to the amino acid sequence (FYWML), not before prolines, under low specificity. Western blot assay was performed according to the protocol described previously (Shah et al. 2017) (see ESM for further details).

\section{Circular dichroism spectroscopy}

CD spectroscopy was used to analyze structural features and thermal stability of the purified VP2 protrusion domain. A small amount of the purified protein $(0.05 \mathrm{mg} / \mathrm{mL}$, volume $300 \mu \mathrm{L})$ in buffer (10 mM potassium phosphate, $\mathrm{pH} 7.4$ ) was transferred into a Hellma quartz cuvette with a path length of $1 \mathrm{~mm}$ for recording CD measurements. Detailed procedures for CD spectroscopy and ThermoFluor assay can be found in the ESM.

\section{Fourier-transform infrared spectroscopy}

FTIR spectroscopy was used to assess the secondary structure and the folding of the purified VP2 protrusion domain. FTIR spectroscopy was performed according to the protocol described elsewhere (Goormaghtigh et al. 1994a, 1994b) (see ESM for further details).

\section{Development of lateral flow immunoassay strips for detection of anti-IBDV antibodies in chickens}

The immunoassay strip is composed of four major components: a cellulose fiber sample pad (Millipore, CFSP203000), a conjugate pad (Millipore, GFCP103000), a NC membrane (Millipore, HF120MC100), and an absorbent pad (Millipore, CFSP173000) (Zhang et al. 2005; Nielsen et al. 2007). A schematic representation of the lateral flow assay is given in Fig. 1. Briefly, the sample pads were saturated in $0.02 \mathrm{M}$ phosphate buffer saline (PBS; $\mathrm{pH}$ 7.4) containing $0.2 \%$ Tween-20 and $1.5 \%$ bovine serum albumin (BSA), dried at $37{ }^{\circ} \mathrm{C}$ for $2 \mathrm{~h}$, and stored at $4{ }^{\circ} \mathrm{C}$ until further use.

\section{Conjugation of the VP2 protrusion domain with gold nanoparticles and development of conjugate pad}

Colloidal gold nanoparticles $(40 \mathrm{~nm})$ were obtained from ClaremontBio. Absorption spectra of gold colloids were measured at 400-700 nm using a UV-visible spectrophotometer (Thermo Fisher Scientific) to verify the monodispersity of nanoparticles. Various amounts of the VP2 protrusion domain $(0,0.2,0.4,0.6,0.8,1.0,1.2$, and $1.6 \mu \mathrm{g})$ were mixed with colloidal gold nanoparticles $\left(200 \mu \mathrm{L} ; A_{530}=6 ; \mathrm{pH} 7.4\right.$ adjusted with $\mathrm{K}_{2} \mathrm{HPO}_{4}(1 \mathrm{M})$ ) and incubated at room temperature for 10-20 min with gentle shaking to produce gold-VP2 protrusion domain conjugates. Then, $10 \% \mathrm{NaCl}(100 \mu \mathrm{L})$ was added to the gold-VP2 protrusion domain conjugate mixture and incubated for $20 \mathrm{~min}$ at room temperature. The samples were monitored for the loss of colloidal stability indicated by the change in color from red to purple/gray. For producing stable gold-VP2 domain, $8 \mu \mathrm{g}$ (optimal amount) of the recombinant protein (VP2 protrusion domain) was added to $1 \mathrm{~mL}$ of colloidal gold solution $\left(A_{530}=6 ; \mathrm{pH} 7.4\right.$ adjusted with $\mathrm{K}_{2} \mathrm{HPO}_{4}(1 \mathrm{M})$ ) and mixed gently for $15 \mathrm{~min}$ in a shaker at room temperature. The admixture was blocked with $10 \% \mathrm{BSA}$ for $1 \mathrm{~h}$ with a brief gentle vortexing and centrifuged at $6000 \times \mathrm{g}$ at $4{ }^{\circ} \mathrm{C}$ for $20 \mathrm{~min}$ to remove unconjugated VP2 protrusion domain. The pellet was suspended in $10 \mathrm{mM}$ sodium phosphate buffer $(\mathrm{pH}$ 7.4) containing $2.5 \%(\mathrm{w} / \mathrm{v})$ sucrose, $1 \%(\mathrm{w} / \mathrm{v}) \mathrm{BSA}$, and $0.05 \%$ sodium azide and stored at $4{ }^{\circ} \mathrm{C}$ until further use. Before development, strips of the conjugate pad were soaked in $10 \mathrm{mM}$ sodium phosphate buffer $(\mathrm{pH} 7.4)$ containing $0.05 \%$ Tween-20 for $12 \mathrm{~h}$ and then dried at $37{ }^{\circ} \mathrm{C}$ for $1-2 \mathrm{~h}$. This was followed by immersion of dried strips of the conjugate pad into conjugate solution until the strips were completely dyed. The wet dyed strips of conjugate pad were then dried at $37{ }^{\circ} \mathrm{C}$ for $2-3 \mathrm{~h}$ on a mesh surface before use or wrapped in an aluminum foil and stored in a desiccator at $4-20{ }^{\circ} \mathrm{C}$. 
Fig. 1 Schematic representation of the lateral flow assay for the detection of anti-IBDV antibodies in chickens. a The components of the strip are shown. $\mathbf{b}$ In the case of a negative sample, only a single red line appears at the control region. $\mathbf{c}$ In the case of a positive sample, two red lines appear: one each at the test and control regions. $\mathrm{C}$ and $\mathrm{T}$ in $\mathbf{b}$ and $\mathbf{c}$ denote the control line and the test line, respectively

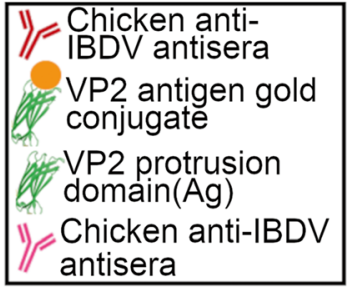

b

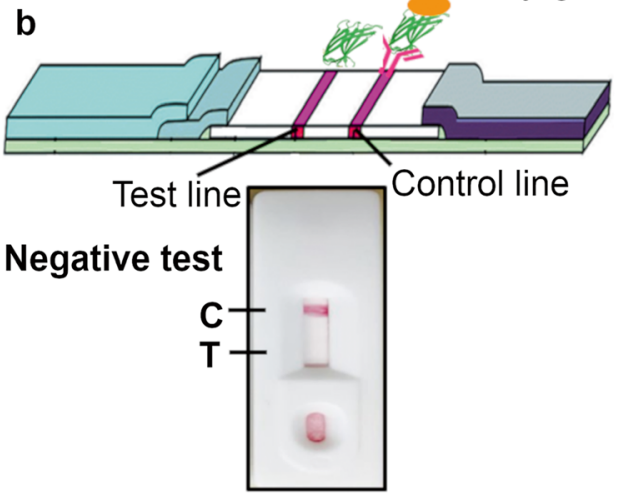

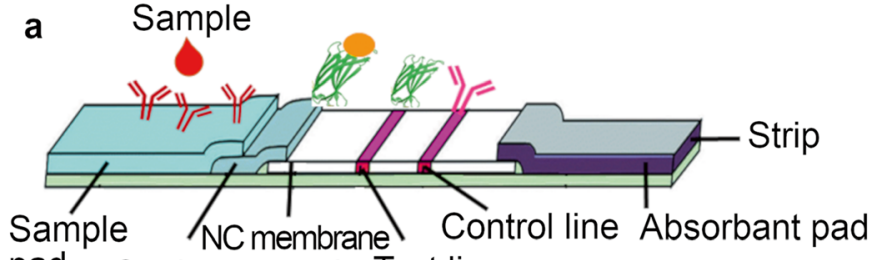

Conjugate pad Test line

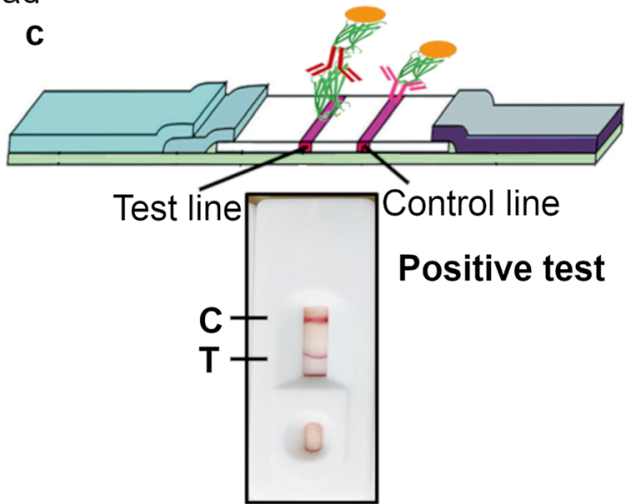

\section{Development of NC membrane, printing of control and test lines, preparation of sample pads, and assembly of the immunoassay strip}

The control line was printed on the NC membrane (Millipore, HF120MC100) with chicken anti-IBDV polyclonal antibodies or anti-VP2 protrusion domain antisera, as per requirement of the experiment. Specific anti-VP2 protrusion domain antisera were obtained upon injection of chickens with the purified recombinant VP2 protrusion domain $(100 \mu \mathrm{g})$ adjuvanted with Montanide ${ }^{\mathrm{TM}}$ ISA 71 VG (1:1) (Seppic, France) at the 7 th and 21 st day $(0.2-\mathrm{mL}$ dose/bird). Blood antisera samples were collected at the 7th, 14th, 21st, and 28th day and were analyzed by enzyme-linked immunosorbent assay (ELISA). Antisera samples collected at the 28th day were used to develop the LFD kit.

The recombinant protein (VP2 protrusion domain; $1 \mathrm{mg}$ / $\mathrm{mL})$ in $10 \mathrm{mM}$ sodium phosphate buffer $(\mathrm{pH}$ 7.4) containing $1 \%$ trehalose or sucrose and $0.09 \%$ sodium azide, respectively, was used to print the test line manually using a pipette of $20-\mu \mathrm{L}$ volume. Both lines (the test line and the control line) were placed $0.5 \mathrm{~cm}$ apart at the center of the $\mathrm{NC}$ membrane. The printed membrane was dried at $37{ }^{\circ} \mathrm{C}$ for $1 \mathrm{~h}$ and then blocked with $5 \mathrm{mM}$ sodium phosphate buffer (pH 7.4) containing $1 \%$ BSA and $0.05 \%$ Tween- 20 . This was followed by 2-3 times washing of the printed NC membrane for 5-10 min with $5 \mathrm{mM}$ sodium phosphate buffer ( $\mathrm{pH}$ 7.4) containing $0.05 \%$ Tween-20. The printed NC membrane was then dried at $37^{\circ} \mathrm{C}$ and stored in a desiccator at $4-20^{\circ} \mathrm{C}$ until further use. Sample pads were treated with $10 \mathrm{mM}$ sodium phosphate buffer (pH 7.4) containing $1 \%$ BSA and $0.05 \%$ Tween-20 for 5-10 min, dried at $37{ }^{\circ} \mathrm{C}$, and stored in a desiccator at 4$20{ }^{\circ} \mathrm{C}$ until further use. The sample pad, the gold conjugated pad, the printed NC membrane, and the absorption pad were assembled sequentially on an adhesive thick card with 1$2 \mathrm{~mm}$ overlapping over each other as shown in Fig. 1. The assembled film was then cut into $0.3-\mathrm{cm}$ pieces, wrapped in an aluminum foil, and stored in a desiccator at $8^{\circ} \mathrm{C}$. The kit was tested with sodium phosphate buffer ( $\mathrm{pH}$ 7.4) and specific pathogen-free (SPF) chicken antisera.

\section{Detection of anti-IBDV antibodies in chickens using gold nanoparticle-based lateral flow assay and commercially available enzyme-linked immunosorbent assay}

For the detection of anti-IBDV antibodies, we have analyzed 10 positive antisera samples from chickens experimentally infected with IBDV (serotype 1), 64 samples from IBDV outbreaks, and 10 uninfected (negative control) serum samples. For obtaining samples from experimentally infected chickens, the experiments were conducted in the Virology Laboratory, School of Animal and Veterinary Sciences, The University of Adelaide, Roseworthy, Australia. Other antisera samples were collected from a local poultry laboratory (Mukhtar Diagnostic Poultry Lab, Samundary Road, Faisalabad, Pakistan). The strip was incubated laterally to allow the sample to pass through the NC membrane for 3-5 min, and the results were recorded. Positive serum samples and negative serum samples (from specific pathogen-free chickens) were analyzed at different dilutions $(5 \times, 10 \times, 20 \times$, and $30 \times)$ prepared using $10 \mathrm{mM}$ sodium phosphate buffer ( $\mathrm{pH}$ 7.4). The specificity of the diagnostic strip toward anti-IBDV antibodies was evaluated against antisera from chickens infected with other poultry viruses. For this purpose, 10 antisera samples of Fowl adenovirus $4(\mathrm{FAdV}-4)$ were tested, produced in our previous 
studies (Shah et al. 2017; Aziz et al. 2019). Antisera samples of infectious bronchitis virus (IBV; 10 samples) and Newcastle disease virus (NDV; 10 samples) were obtained from a local poultry laboratory (Mukhtar Diagnostic Poultry Lab, Samundary Road, Faisalabad, Pakistan) during the natural disease outbreaks. For the assessment of the storage life, the anti-IBDV antibody detection strips were sealed in aluminum foil bags with a desiccant and placed at $8{ }^{\circ} \mathrm{C}$. The efficiency of diagnostic strips was tested by analyzing the antisera of IBDV-infected chickens after every 3 months.

For comparison, the same anti-IBDV sera samples were analyzed using a commercially available ELISA kit (IDEXX IBD-Ab test, Westbrook, USA), according to the manufacturer's instructions. Briefly, anti-IBDV serum samples $(100 \mu \mathrm{L})$ were applied on an antigen (IBDV)-coated 96-well plate and incubated with the supplied conjugate and $3,3^{\prime}, 5,5^{\prime}$ tetramethylbenzidine (TMB) substrate $(100 \mu \mathrm{L}$ each) to develop the signal. The absorbance at $650 \mathrm{~nm}$ was recorded using an ELISA microplate reader.

\section{Results}

\section{Selection of the protrusion domain of VP2 protein as a highly immunogenic region}

VP2 is a common capsid protein of birnaviruses (Azad et al. 1987; Böttcher et al. 1997; Pous et al. 2005; Letzel et al. 2007; Delmas et al. 2012; Taghavian et al. 2013; Wang et al. 2019). The VP2 capsid protein of IBDV, a member of Birnaviridiae family, has been reported to be highly immunogenic (Azad et al. 1987; Letzel et al. 2007; Taghavian et al. 2013; Wang et al. 2019). Considering the immunogenic nature of the protein, the three-dimensional structure of the VP2 protein was analyzed to identify potential epitopic regions. The VP2 crystal structure (PBD code 2df7) comprises three well-folded domains: a protrusion domain, a shell, and a base domain (Supplementary Fig. S1) (Lee et al. 2006). Among these, the VP2 protrusion domain containing multiple loops is located on the capsid surface which covers the major immunodominant antigenic domain (Asp201-Gly339); therefore, it was selected for testing as a promising immunogenic region for the diagnosis of anti-IBDV antibodies in chickens.

\section{Amplification of the gene encoding the VP2 protrusion domain from a local IBDV strain and construction of expression vector}

The local vvIBDV strain was confirmed by reverse transcriptase PCR which produced a 743-bp fragment corresponding to the VP2 gene of vvIBDV (Supplementary Fig. S2). A nucleotide sequence of 417 bp encoding the VP2 protrusion domain was amplified by PCR using the
cDNA of vvIBDV as a template (Supplementary Fig. S3). The gene segment corresponding to the VP2 protrusion domain was cloned into pET28a vector which allowed the expression of the VP2 protrusion domain fused to an oligo-histidine $\left(\mathrm{His}_{6}\right)$ tag and a PreScission (HRV 3C) protease recognition site at the $\mathrm{N}$-terminus (Ullah et al. 2016). The resultant expression vector was named pET28a-PreS-VP2 protrusion domain (Supplementary Fig. S4). DNA sequencing results confirmed the integrity of the VP2 protrusion domain and the correct reading frame of the cloned sequence in the vector. The nucleotide sequence of the amplified gene was found to be identical to the sequence of IBDV-PK-1 VP2 gene (GenBank accession number KT281984.1).

\section{Heterologous expression, purification, and identification of the recombinant VP2 protrusion domain}

The recombinant VP2 protrusion domain was successfully expressed in E. coli BL21 (DE3) cells. The overexpressed domain in the soluble form was purified using nickelaffinity chromatography to more than $95 \%$ purity in quantities sufficient for structural studies and antibody detection (Fig. 2a). The obtained yield of the VP2 protrusion domain was $10 \mathrm{mg} / \mathrm{L}$ culture volume. The sodium dodecyl sulphate-polyacrylamide gel electrophoresis (SDS-PAGE) analysis showed that the purified domain migrates in three oligomeric forms corresponding to apparent molecular weights of $\sim 13 \mathrm{kDa}, \sim 23 \mathrm{kDa}$, and $\sim 35 \mathrm{kDa}$, representing monomeric, dimeric, and trimeric forms of the protein, respectively, and suggesting that higher oligomers were SDS- $\beta$ ME-heat resistant. The identity of these oligomer forms was confirmed by western blotting using anti-His 6 antibodies (Fig. 2b). The apparent faster migration of the oligomers (monomers, dimmers, and trimers) on the SDSPAGE gel compared with their calculated molecular weights $(17 \mathrm{kDa}, 34 \mathrm{kDa}$, and $51 \mathrm{kDa})$ can be attributed to the relatively high hydrophobic content $(70 \%)$ of the domain as predicted by hydropathy analysis (http://www. ebi.ac.uk/Tools/seqstats/emboss_pepinfo) (Fig. 2c) (Kyte and Doolittle 1982). Such migration behavior is usually displayed by proteins that contain a higher proportion of hydrophobic amino acids and is commonly observed in the case of membrane proteins (Rahman et al. 2007).

To confirm the identity of the full-length VP2 protrusion domain by MS, the protein was obtained in a highly purified form after gel filtration (Fig. 2d, e). The MS analysis confirmed that the purified oligomers represented the full-length domain. Furthermore, digestion with chymotrypsin generated nine peptides, covering the entire VP2 protrusion domain (Fig. 2f). 

$\begin{array}{llllllllll}M & 1 & 2 & 3 & 4 & 5 & 6 & 7 & 8 & 9\end{array}$
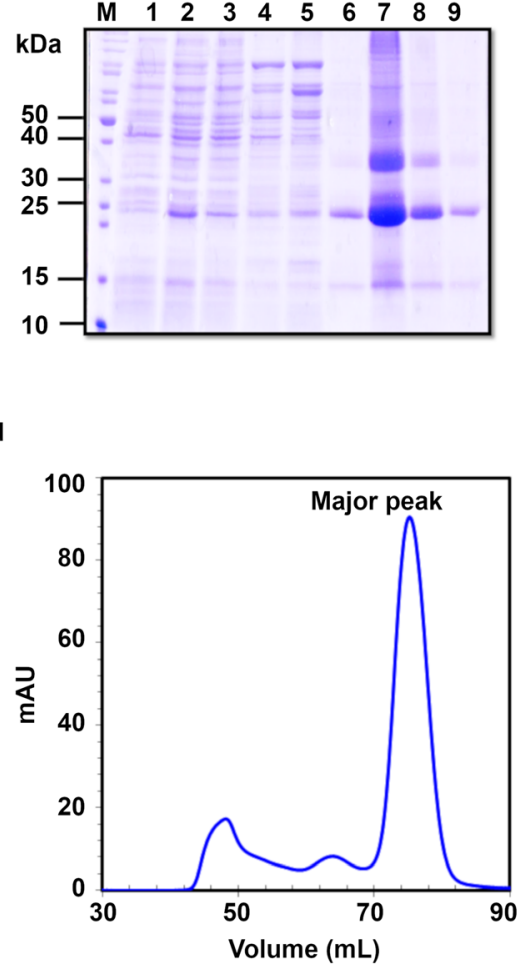
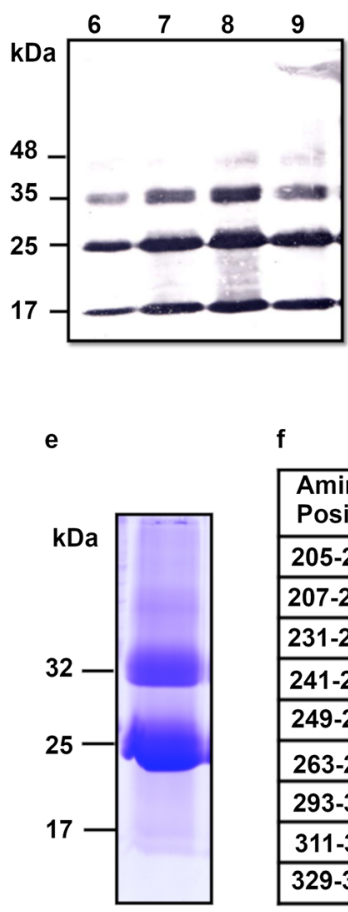

f

\begin{tabular}{|l|l|l|}
\hline $\begin{array}{l}\text { Amino Acid } \\
\text { Position }\end{array}$ & Mass (Da) & Sequence \\
\hline $205-220$ & 1871.83 & VYTITAADDYQFSSQY \\
\hline $207-230$ & 2627.25 & TITAADDYQFSSQYQTGGVTITLF \\
\hline $231-244$ & 1018.58 & SAKIDAITSL \\
\hline $241-255$ & 1534.81 & SIGGELVFQTSVQGL \\
\hline $249-262$ & 1463.81 & QTSVQGLILGATIY \\
\hline $263-292$ & 3067.54 & LIGFDGTTVITRAVAADNGLTAGTDNLMPF \\
\hline $293-310$ & 1976.14 & NIVIPTSEITQPITSIKL \\
\hline $311-327$ & 1796.81 & EIVTSKSGGQAGDQMSW \\
\hline $329-339$ & 1012.54 & ASGSLAVTIHG \\
\hline
\end{tabular}

Fig. 2 Expression, purification, western blot, hydropathy, and MS analyses of $\mathrm{His}_{6}$-PreS-VP2 protrusion domain in the $E$. coli strain BL21 (DE3). a Lanes 1, 2, and 3 show the unbound protein fraction, the pellet of expressed cells, and the supernatant after cell lysis, respectively. Lanes 4 and 5 show wash fractions, and the purified protein (various oligomeric forms) obtained after elution has been shown in lanes 6-9. The mobilities of molecular weight markers (Bio Basic Cat \# BSM0661) are shown in lane M. b Purified VP2 protrusion domain (corresponding to lanes 6-9 on the SDS-PAGE gel) was confirmed by western blot using anti-histidine antibodies. c Hydropathy plot predicts that the VP2 protrusion domain is predominantly hydrophobic $(70 \%)$, suggesting faster migration of the protein on the SDS-PAGE gel compared with the actual molecular weight of various oligomers. Amino acid numbers of the domain are assigned

\section{Structural and thermal stability analysis of the recombinant VP2 protrusion domain}

The structural characterization of the VP2 protrusion domain was performed using CD and FTIR spectroscopic techniques. The results suggested that the domain was properly folded and contained $\beta$-sheets as the predominant secondary structure element. The CD spectra of the VP2 protrusion domain and potassium phosphate buffer ( $\mathrm{pH}$ 7.4) are shown in Fig. 3a. The CD spectrum of the VP2 protrusion domain obtained after subtraction from the spectrum of potassium phosphate buffer $(\mathrm{pH} 7.4)$ revealed mixed secondary structure elements that are represented by characteristic minima around $220 \mathrm{~nm}$ and maxima at $195 \mathrm{~nm}$ (Fig. 3b) (Kelly et al. 2005). The overall quantitative estimation of the secondary structure content showed $\sim 42 \% \beta$-sheet, $\sim 23 \% \alpha$-helices, $\sim 18 \% \beta$-turns/bends, and $17 \%$ unordered regions in the protrusion domain (Table 1). corresponding to their positions in the VP2 protein. $\mathbf{d}$ Gel filtration of the VP2 protrusion domain using a Superdex 75 16/60 GL column. e The major peak fraction of the VP2 protrusion domain from gel filtration was electrophoresed on a $12 \%$ SDS-PAGE gel which shows that the trimeric form cannot be completely resolved by SDS- $\beta$ ME-heat. $\mathbf{f}$ The peptide fragments identified by mass fingerprinting shown in increasing amino acid order confirmed the identity of the full-length domain in each band extracted from the SDS-PAGE gel. The peptide identification was performed through the use of a high mass accuracy scan to determine the mass of the intact peptide and the fragmentation of protein (MS/MS). A reverse database with a very low threshold value for false discovery rate (1-2\%) was used to filter the resulting peptide data for the best matched sequences

The thermal assay was performed to investigate the stability of the folded conformation of the recombinant protrusion domain. Changes in CD ellipticity were monitored for the protein (gradually cooled down to room temperature) after heating to various temperatures $\left(50{ }^{\circ} \mathrm{C}, 70{ }^{\circ} \mathrm{C}\right.$, and $\left.90{ }^{\circ} \mathrm{C}\right)$. The analysis revealed that the $\mathrm{CD}$ spectrum was almost unaltered for the domain heated to $50^{\circ} \mathrm{C}$ and reproduced the same minima and maxima as the non-heated protein $\left(25^{\circ} \mathrm{C}\right)$. Exceeding this temperature, there was a significant decrease observed in the CD ellipticity, indicating that the domain loses its folded conformation at temperatures higher than $50{ }^{\circ} \mathrm{C}$ (Fig. 3c). Furthermore, to verify the thermal stability of the domain, ThermoFluor assay was performed and data were analyzed using the CFX Manager software (Rosa et al. 2015). The resulting sigmoidal thermal shift curve showed that the recombinant domain retained its folded conformation up to $50{ }^{\circ} \mathrm{C}$. The melting temperature (Tm) of $60^{\circ} \mathrm{C}$ obtained 
a

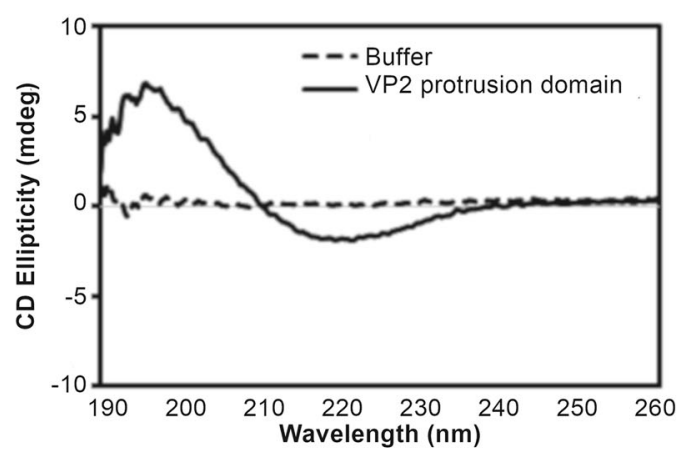

c

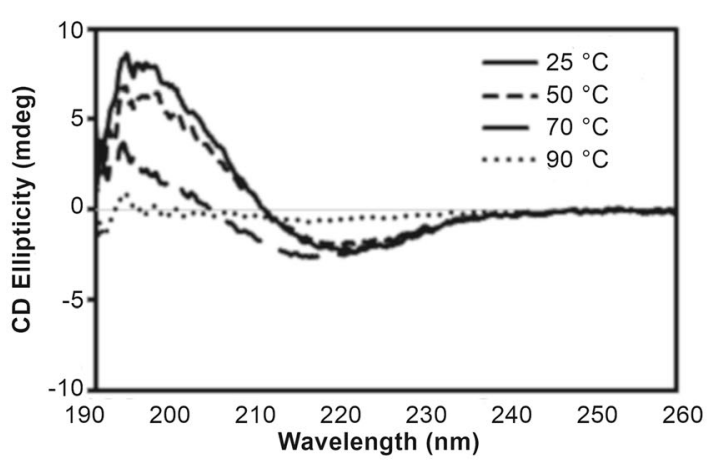

e

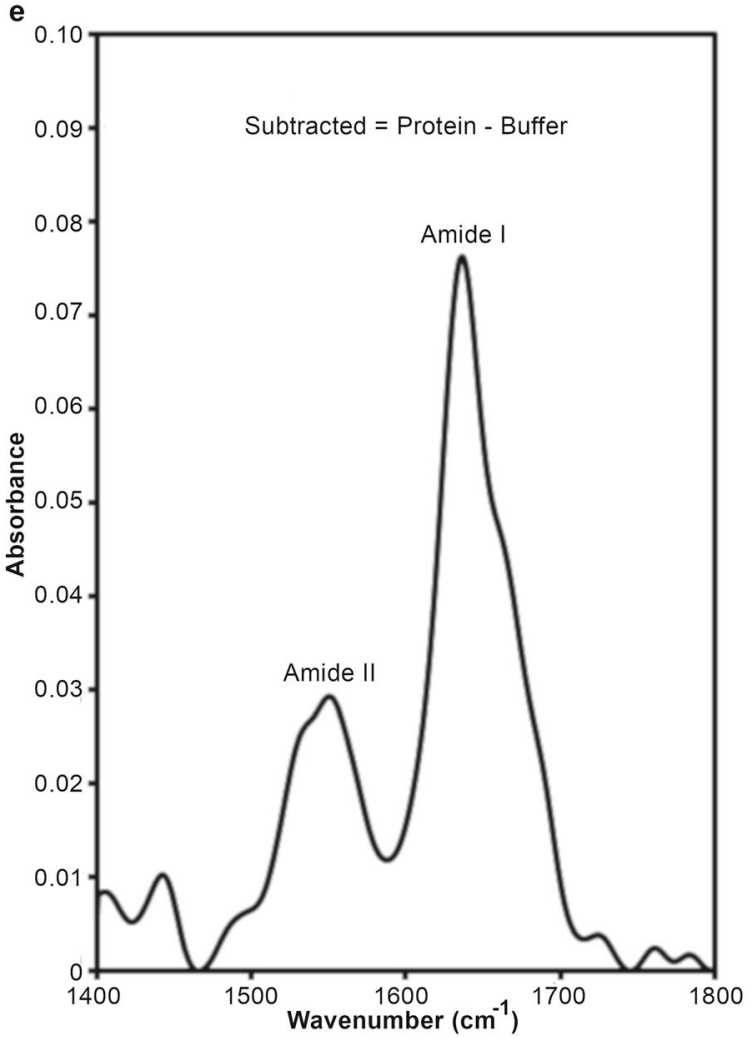

Fig. 3 Characterization of $\mathrm{His}_{6}-\mathrm{PreS}-\mathrm{VP} 2$ protrusion domain by $\mathrm{CD}$, FTIR spectroscopy, and ThermoFluor assay. a CD spectra of the VP2 protrusion domain (continuous line) and potassium phosphate buffer, $\mathrm{pH} 7.4$, (dotted line) have been shown separately. b The VP2 protrusion domain spectra subtracted from potassium phosphate buffer, $\mathrm{pH}$ 7.4. c Thermal stability analysis of $\mathrm{His}_{6}$-PreS-VP2 protrusion domain by $\mathrm{CD}$ spectroscopy shows that the protein is properly folded and thermostable at $\sim 50{ }^{\circ} \mathrm{C}$. d Thermal shift curve of $\mathrm{His}_{6}$-PreS-VP2 protrusion domain

b

d

f
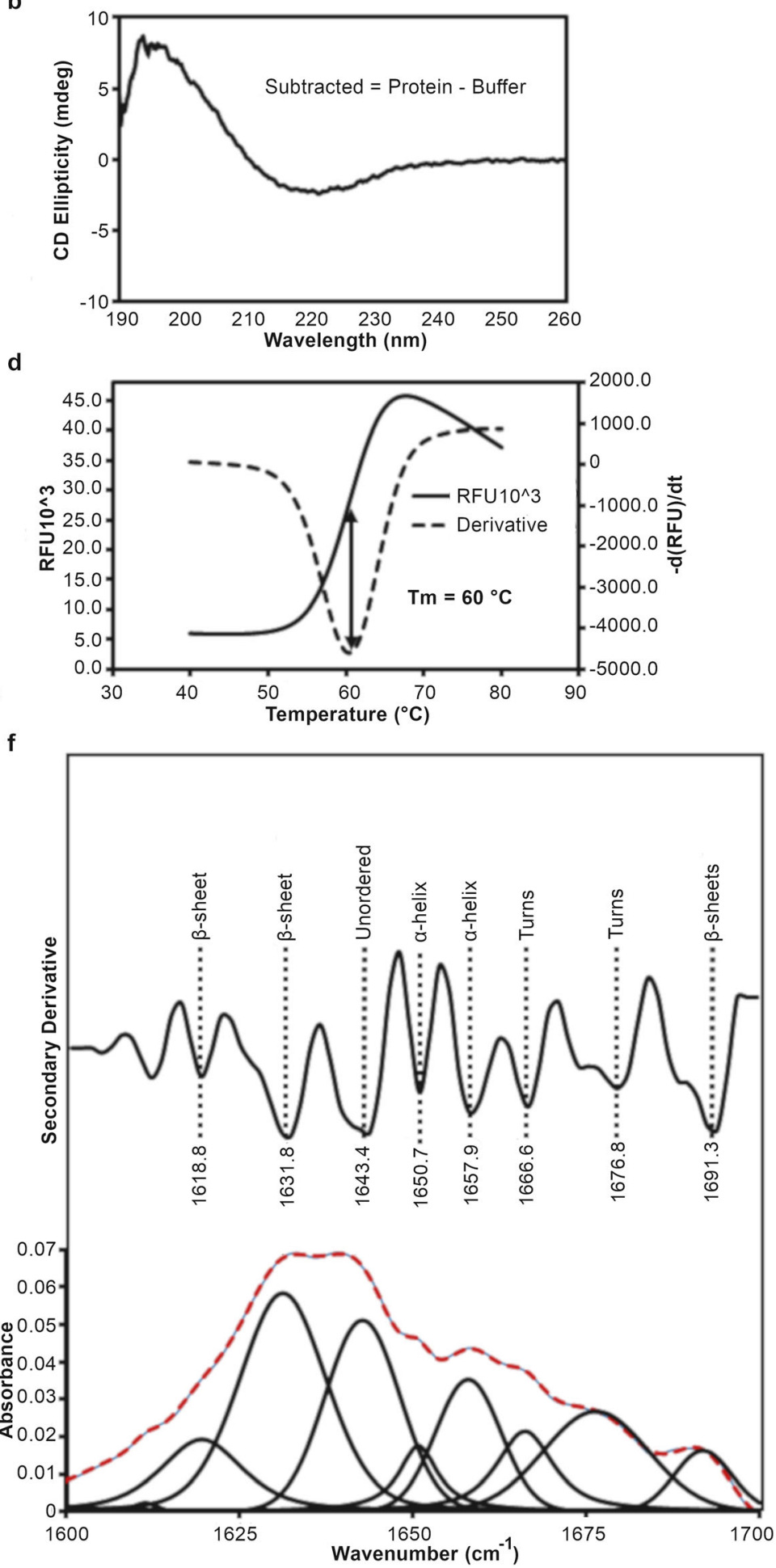

showing the melting temperature $\left(\mathrm{Tm}=60{ }^{\circ} \mathrm{C}\right)$ at which $50 \%$ of the protein is unfolded. The dotted line represents the first derivative of fluorescence emission as a function of temperature [-d(RFU)/dT]. e The FTIR spectrum of the VP2 protrusion domain obtained by subtraction from the spectrum of Tris-Cl buffer ( $\mathrm{pH} 7.8)$. f Secondary derivative (upper panel) and deconvolution of amide I region (lower panel) of the FTIR spectrum of the VP2 protrusion domain, and retrieval of individual bands. The dotted red line shows the curve fitted using component bands 
Table 1 Secondary structure analysis of the VP2 protrusion domain by FTIR and CD

\begin{tabular}{lllll}
\hline Techniques & $\alpha$-helices (\%) & $\beta$-sheets (\%) & $\beta$-turns/bends (\%) & Unordered (\%) \\
\hline FTIR $^{\mathrm{a}}$ & 17 & 42 & 22 & 19 \\
$\mathrm{CD}^{\mathrm{b}}$ & 23 & 42 & 18 & 17 \\
\hline
\end{tabular}

${ }^{\text {a }}$ For FTIR, band assignment for interpretation of spectra (shown in Fig. 3e) was made on the basis of previous measurements (Goormaghtigh et al. 1994a, 1994b)

${ }^{\mathrm{b}} \mathrm{CD}$ spectrum was analyzed using DichroWeb server (Whitmore and Wallace 2004, 2008) developed for structural analysis of proteins as discussed in Methods section at the midpoint of the curve and at the apex of the 1st derivative depicts partial unfolding of the protein (Fig. 3d).

The FTIR spectrum contains representative amide I and II peaks in the $1500-1700 \mathrm{~cm}^{-1}$ region which is one of the characteristics of folded proteins (Fig. 3e). The interpreted 2nd derivative data (Origin graphic software) (Wass 2002) belonging to amide I peak confirmed the presence of secondary structure elements in the protrusion domain (Fig. 3f, upper panel). To estimate the secondary structure content, the amide I (1600$1700 \mathrm{~cm}^{-1}$ ) region was reproduced by a fit of eight components which suggested a higher proportion of $\beta$-sheet contents in the domain (Fig. 3f, lower panel). The quantitative analysis of the FTIR spectrum predicted $\sim 42 \% \beta$-sheets, $\sim 17 \% \alpha$-helices, $22 \% \beta$-turns/bends, and $\sim 19 \%$ unordered regions, similar to that obtained from the $\mathrm{CD}$ analysis (Table 1).

\section{Detection of anti-IBDV antibodies using gold nanoparticle-based lateral flow assay and commercially available enzyme-linked immunosorbent assay}

The absorbance ratio $\left(A_{650} / A_{530}=0.09\right)$ of gold nanoparticles as determined by visible spectrophotometry confirmed that the particles were monodisperse and free of aggregates (Fig. 4a). The optimal concentration of the VP2 protrusion domain for conjugation with gold nanoparticles was $1.6 \mu \mathrm{g}$ per $200 \mu \mathrm{L}(8 \mu \mathrm{g} / \mathrm{mL})$ of gold suspension in $10 \% \mathrm{NaCl}$ (Fig. 4b). The resultant concentration of the protein was used for generation of colloidal gold nanoparticles-VP2 protrusion domain (antigen) conjugates, and the strip was assembled for the detection assay (as described in the "Materials and methods" section).

The sensitivity of the strip was evaluated by applying different dilutions $(5 \times, 10 \times, 20 \times$, and $30 \times)$ of known IBDV anti-sera. For comparison, a negative sample (consisting of $10 \mathrm{mM}$ sodium phosphate buffer $(\mathrm{pH} 7.4)$ and antisera isolated from the SPF chicken $(5 \times)$ ) was also applied to the sample pad. In the case of positive anti-IBDV samples, two red lines appeared: one at the test region and the other at the control region. In contrast, only a single line appeared at the control region in the case of negative samples (Supplementary Fig. S5). These results confirmed the presence of anti-IBDV antibodies in serum samples of chickens, suggesting that the strip could successfully be applied for diagnosis of anti-IBDV antibodies in chickens. In the absence of specific antibodies, sometimes due to the (non-specific) interaction of VP2-gold nanoparticle conjugates with proteins from the serum, large aggregates termed as clouds or corona are formed (de Puig et al. 2017) which interfere with the signal intensity at the control line. Therefore, various dilutions $(10 \times, 20 \times, 30 \times$, and $100 \times)$ of antisera isolated from SPF-chickens were tested in order to get the best signal, and signals with improved quality were observed in the diluted SPF-antisera, likely due to less non-specific proteins (Supplementary Fig. S5a, b). We find that the signal intensity at the control line increases when antisera raised against the VP2 protrusion domain are printed instead of anti-IBDV antisera at the control line (Supplementary Fig. S5c).

The analysis of different test samples collected from fields having frequent IBDV outbreaks revealed that the samples were positive, confirming the presence of anti-IBDV antibodies (Fig. 4c). The efficiency of the IBD diagnostic strips (sealed in aluminum foil with silica beads desiccant and stored at $8{ }^{\circ} \mathrm{C}$ ) was assessed periodically after an interval of 3 months, yielding consistent results for 21 months, tested so far (Fig. 4d).

To check the specificity of the lateral flow diagnostic strip, different antisera samples from chickens infected with FAdV-4, IBV, and NDV were tested, which showed no cross-reactivity with other common antibodies present in chickens.

The developed LFD yields comparable results as obtained through a commercial ELISA (IBD IDEXX) kit. The cutoff value for the antibody titer of the commercial ELISA kit is 396 , and a serum containing a similar amount of antibodies (titer 500) can be used to produce a signal at the test line in the developed LFD kit, indicating that the LFD can be equally used to detect in qualitative terms the anti-IBDV antibodies in infected chickens. In excellent agreement with results obtained from the ELISA kit, our developed LFD yielded $100 \%$ sensitivity, $100 \%$ specificity, $100 \%$ positive predictive value, $100 \%$ negative predictive value, and Youden's index equal to 1 , suggesting that the LFD kit can be reliably used for the detection of anti-IBDV antibodies in the serum samples of chickens in a qualitative manner. 


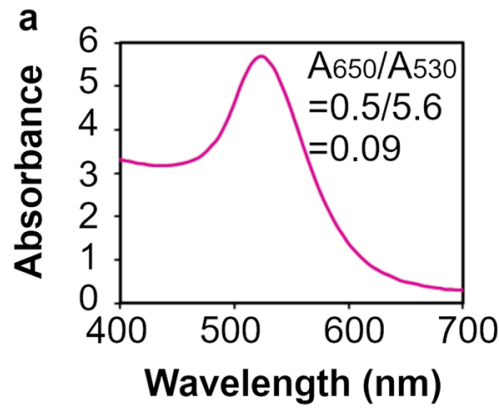

C

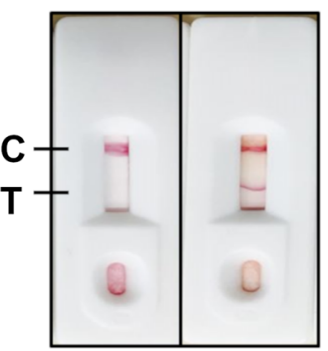

d b

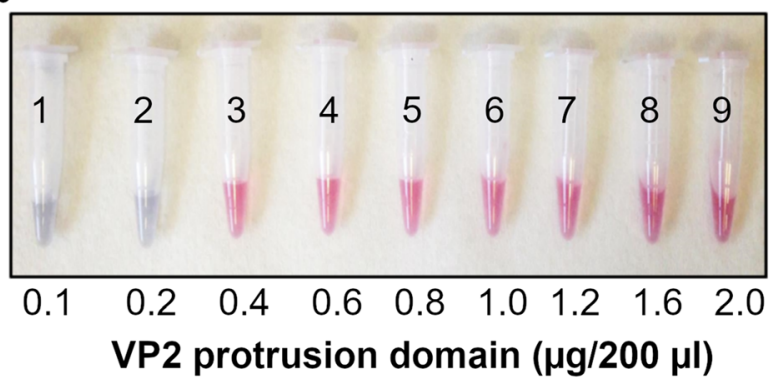

d

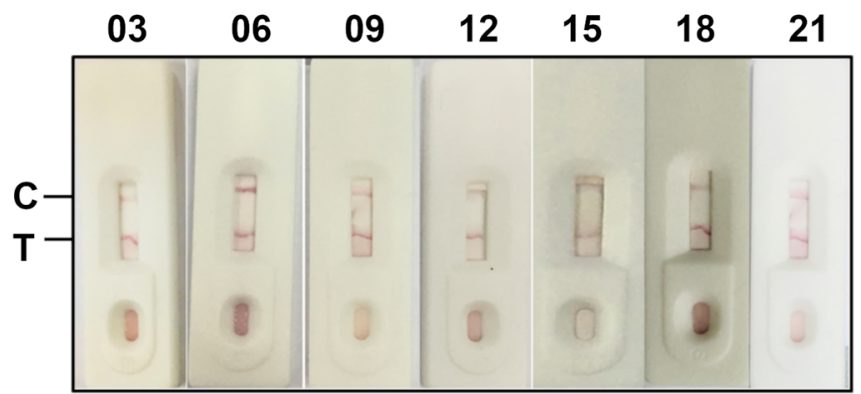

Fig. 4 Absorption scan for determining the stability of gold nanoparticles, detection of anti-IBDV antibodies of infected chickens, and time-based analysis of the antisera. a The ratio, $\mathrm{A}_{650} / \mathrm{A}_{530}(0.5 / 5.6)$, of 0.09 obtained during the absorption scan $(400-700 \mathrm{~nm})$ of the gold nanoparticles shows that the particles are monodisperse. $\mathbf{b}$ The stable red color in tubes 8 and 9 indicates that a protein concentration of $8 \mu \mathrm{g} / \mathrm{mL}$ or above is sufficient to produce stable colloidal gold-antigen conjugates. $\mathbf{c}$
Detection assay shows the presence of anti-IBDV antibodies in the serum of the positive sample (right), indicated by the appearance of a red line at the test region, compared with the negative sample (left). d The timebased analysis of the antisera from infected chickens by the diagnostic strip shows that the strip can be used to detect successfully anti-IBDV antibodies up to 21 months (tested periodically every 3 months)

birnaviruses share a similar overall fold (Supplementary Fig. S6). Capsid protein-based lateral flow assays have recently received increased attention due to their ease-of-use and cost-effectiveness (Sun et al. 2014; Sastre et al. 2016). A capsid protein-based lateral flow assay is currently not available for birnaviruses. In this study, the VP2 protrusion domain (Asp201-Gly339) was selected as a diagnostic antigen since it is exposed on the surface of the protein and protrudes out of the viral capsid, suggesting that the domain can act as an epitopic region (Vakharia et al. 1994; Lee et al. 2006). Furthermore, different studies have revealed that proteinprotein interactions that lead to cellular functions such as signaling, regulation, and antibody neutralization are mediated through surface loops of proteins (Drakopoulou et al. 1996; Osajima and Hoshino 2016).

The VP2 protrusion domain was heterologously expressed in a soluble form in E. coli and purified through chromatographic techniques. To the best of our knowledge, the recombinant VP2 protrusion domain heterologously expressed in prokaryotic or eukaryotic systems has not been used in a lateral flow assay yet. The associated advantage with the heterologous expression of the VP2 protrusion domain is that the protein can be produced in sufficient amounts readily and cost-effectively, allowing the diagnostic capacity of the lateral flow assay to be easily scaled up for large flocks. However, the proper folding of disease in chickens. The VP2 protrusion domain is found in genera of Birnaviridae family, and domains from different 
recombinant proteins produced in heterologous expression systems is a major key point for successfully conducting structural and functional studies. A protein produced in the soluble fraction may not always be folded into a stable secondary structure conformation compared with the native protein structure. Therefore, to produce an authentic protein for practical applications such as for use in the lateral flow assay, biophysical studies of the VP2 protrusion domain were performed using $\mathrm{CD}$ and FTIR spectroscopic techniques. These studies revealed that the domain is properly folded containing a high proportion of $\beta$-sheet secondary structures similar to the native VP2 protrusion domain (PDB code 2df7) (Lee et al. 2006). For field applications, the stability of recombinant proteins is of utmost importance in diverse environmental conditions. Therefore, the folded conformation of the VP2 protrusion domain was evaluated at elevated temperatures using CD spectroscopy and ThermoFlour assay. The domain was found structurally stable and remained properly folded at $50{ }^{\circ} \mathrm{C}$, suggesting that the protein could be used for a wide range of field applications on the basis of its thermal stability. Similar to the native form of the VP2 full-length protein, the VP2 protrusion domain exists as a trimer (Azad et al. 1987; Letzel et al. 2007; Taghavian et al. 2013; Wang et al. 2019). It has been reported previously that the oligomeric state of the full-length VP2 protein is stabilized upon binding of calcium ions (Lee et al. 2006). Interestingly, the domain is devoid of the calcium binding site, even then it exists in a trimeric state. These characteristics, combined with sufficiently high thermostability, render the purified VP2 protrusion domain an attractive target for use as a diagnostic antigen in immunoassays.

For the lateral flow assay, the purified VP2 protrusion domain was conjugated with colloidal gold nanoparticles $(40 \mathrm{~nm})$ for the detection of anti-IBDV antibodies in the serum samples of infected chickens. The reactivity criteria were analyzed by the appearance of red lines at test and control regions within 3-5 min after addition of the serum sample. The gold nanoparticle-based lateral flow assay can efficiently detect anti-IBDV antibodies in antisera samples from infected chickens which were confirmed as positive antisera samples using a commercially available ELISA kit, suggesting that the reported assay yields comparable qualitative results as obtained through ELISA. However, the ELISA is a highly sensitive method for the detection of anti-IBDV antibodies (Dey et al. 2009; Singh et al. 2010), but here, we have developed a lateral flow assay based on VP2 protrusion domain which is a rapid on-site diagnostic test for the detection of anti-IBDV antibodies in infected chickens. Compared with ELISA, which needs laboratory equipment and trained personnel, the lateral flow assay is a fast, easy, and cost-effective technique for the diagnosis of anti-IBDV antibodies. To monitor the pathogenic IBDV in the infected chickens, previously, a one-step antiIBDV antibody strip was developed for the detection of the virus from the bursa samples (Zhang et al. 2005). In our study, we have developed LFD based on the VP2 protrusion domain for the detection of the anti-IBDV antibodies of serotype 1 in the serum samples was conducted using antisera collected from the blood of infected chickens. Due to a highly stable nature of the VP2 protrusion domain, the developed LFD kit has a shelf-life of 21 months, tested so far.

As VP2 is a common capsid protein of birnaviruses (Azad et al. 1987; Böttcher et al. 1997; Pous et al. 2005; Letzel et al. 2007; Delmas et al. 2012; Taghavian et al. 2013; Wang et al. 2019), the lateral flow assay reported here can be generalized for detection of anti-birnaviral antibodies in serum samples of animals infected with other birnaviruses. Furthermore, the assay can be adapted for the detection of members of Tetraviridae and Nodaviridae families which contain homologous VP2 capsid proteins. Furthermore, the surface-exposed VP2 protrusion domain can be exploited as a vaccine candidate.

In conclusion, the protrusion domain of a birnaviral capsid protein, VP2, was successfully employed as a diagnostic antigen for the development of a lateral flow assay. The biophysical characterization of the purified domain reveals that the domain remains folded at high temperatures, making it suitable for diagnostic purposes. The lateral flow assay was used to successfully detect antibodies specific to IBDV in the serum samples of infected chickens during laboratory testing and onsite testing. Similar to a commercial ELISA, the lateral flow assay reported here allows the detection of anti-IBDV antibodies in serum samples of infected chickens and has a shelf-life of 21 months tested so far. The lateral flow assay can be generalized for the detection of other birnaviruses and members of Tetraviridae and Nodaviridae families which contain homologous VP2 capsid proteins.

Acknowledgements We thank SSE-LUMS, Pakistan, and Elettra Synchrotron facility, Italy, for access to FTIR and CD spectrometers, respectively.

Availability of data and materials All data and materials are available from the corresponding authors on reasonable request.

Authors' contributions Mudasser Habib, Muhammad Muddassar, Mazhar Iqbal, Farhid Hemmatzadeh, and Moazur Rahman conceived the experiments. Maryam Zafar, Majid Ali Shah, Anam Tariq, Mudasser Habib, Muhammad Salahuddin Shah, Farhid Hemmatzadeh, and Moazur Rahman conducted the experiments. Maryam Zafar, Majid Ali Shah, Aamir Shehzad, Anam Tariq, Mudasser Habib, Muhammad Muddassar, Muhammad Salahuddin Shah, Mazhar Iqbal, Farhid Hemmatzadeh, and Moazur Rahman analyzed the results. Maryam Zafar, Majid Ali Shah, Aamir Shehzad, Anam Tariq, Muhammad Muddassar, Muhammad Salahuddin Shah, Farhid Hemmatzadeh, and Moazur Rahman wrote the manuscript. All authors reviewed the manuscript.

Funding information This work was supported in part by the Higher Education Commission (HEC) of Pakistan (TDF grant No. 003-006; awarded to Moazur Rahman), HEC-IRSIP Fellowship (awarded to Maryam Zafar), and International Atomic Energy Fellowship (awarded to Moazur Rahman). 


\section{Compliance with ethical standards}

Competing interests The authors declare that they have no competing interests.

Ethics approval and consent to participate This work has received approval for research ethics from the Institutional Animal Care and Use Committee (IACUC), Animal Sciences Division, Nuclear Institute for Agriculture and Biology (NIAB), Faisalabad, Pakistan. The experiments were performed following the guidelines of the IACUC of NIAB, Faisalabad, Pakistan. This article does not contain any studies with human participants performed by any of the authors.

\section{References}

Azad AA, Jagadish MN, Brown MA, Hudson PJ (1987) Deletion mapping and expression in Escherichia coli of the large genomic segment of a birnavirus. Virology 161:145-152. https://doi.org/10. 1016/0042-6822(87)90180-2

Aziz F, Tufail S, Shah MA, Shah MS, Habib M, Mirza O, Iqbal M, Rahman M (2019) In silico epitope prediction and immunogenic analysis for penton base epitope-focused vaccine against hydropericardium syndrome in chicken. Virus Res 273:197750. https://doi.org/10.1016/j.virusres.2019.197750

Banda A, Villegas P, El-Attrache J (2003) Molecular characterization of infectious bursal disease virus from commercial poultry in the United States and Latin America. Avian Dis 47:87-96. https://doi. org/10.1637/0005-20862003047

Bayliss CD, Spies U, Shaw K, Peters RW, Papageorgiou A, Muller H, Boursnell MEG (1990) A comparison of the sequences of segment A of four infectious bursal disease virus strains and identification of a variable region in VP2. J Gen Virol 71(Pt 6):1303-1312. https:// doi.org/10.1099/0022-1317-71-6-1303

Böttcher B, Kiselev NA, Stel'Mashchuk VY, Perevozchikova NA, Borisov AV, Crowther RA (1997) Three-dimensional structure of infectious bursal disease virus determined by electron cryomicroscopy. J Virol 71:325-330

Castón JR, Martínez-Torrecuadrada JL, Maraver A, Lombardo E, Rodriguez JF, Casal JI, Carrascosa JL (2001) C terminus of infectious bursal disease virus major capsid protein VP2 is involved in definition of the T number for capsid assembly. J Virol 75:1081510828. https://doi.org/10.1128/JVI.75.22.10815-10828.2001

Chettle N, Stuart JC, Wyeth PJ (1989) Outbreak of virulent infectious bursal disease in East Anglia. Vet Rec 125:271-272. https://doi.org/ 10.1136/vr.125.10.271

Cosgrove AS (1962) An apparently new disease of chickens: avian nephrosis. Avian Dis 6:385. https://doi.org/10.2307/1587909

De Puig H, Bosch I, Gehrke L, Hamad-Schifferli K (2017) Challenges of the nano-bio interface in lateral flow and dipstick immunoassays. Trends Biotechnol 35:1169-1180. https://doi.org/10.1016/j.tibtech. 2017.09.001

Delmas B, Mundt E, Vakharia VN, Wu JL (2012) Birnaviridae. In: Virus Taxonomy Elsevier, pp 499-507

Dey S, Upadhyay C, Madhan Mohan C, Mohan Kataria J, Vakharia VN (2009) Formation of subviral particles of the capsid protein VP2 of infectious bursal disease virus and its application in serological diagnosis. J Virol Methods 157:84-89. https://doi.org/10.1016/j. jviromet.2008.11.020

Drakopoulou E, Zinn-Justin S, Guenneugues M, Gilqin B, Menez A, Vita C (1996) Changing the structural context of a functional beta-hairpin. Synthesis and characterization of a chimera containing the curaremimetic loop of a snake toxin in the scorpion alpha/beta scaffold. J Biol Chem 271:11979-11987. https://doi.org/10.1074/ jbc.271.20.11979

Fahey KJ, Erny K, Crooks J (1989) A conformational immunogen on VP-2 of infectious bursal disease virus that induces virusneutralizing antibodies that passively protect chickens. J Gen Virol 70:1473-1481. https://doi.org/10.1099/0022-1317-70-6-1473

Goormaghtigh E, Cabiaux V, Ruysschaert JM (1994a) Determination of soluble and membrane protein structure by Fourier transform infrared spectroscopy. III. Secondary structures. Subcell Biochem 23: 405-450. https://doi.org/10.1007/978-1-4615-1863-1

Goormaghtigh E, Cabiaux V, Ruysschaert JM (1994b) Determination of soluble and membrane protein structure by Fourier transform infrared spectroscopy. I. Assignments and model compounds. Subcell Biochem 23:329-362. https://doi.org/10.1007/978-1-4615-1863-1_

Heine HG, Haritou M, Failla P, Fahey K, Azad A (1991) Sequence analysis and expression of the host-protective immunogen VP2 of a variant strain of infectious bursal disease virus which can circumvent vaccination with standard type I strains. J Gen Virol 72:18351843. https://doi.org/10.1099/0022-1317-72-8-1835

Hill BJ, Way K (1995) Serological classification of infectious pancreatic necrosis (IPN) virus and other aquatic birnaviruses. Annu Rev Fish Dis 5:55-77. https://doi.org/10.1016/0959-8030(95)00011-9

Jackwood DJ, Nielsen CK (1997) Detection of infectious bursal disease viruses in commercially reared chickens using the reverse transcriptase/polymerase chain reaction-restriction endonuclease assay. Avian Dis 41:137-143. https://doi.org/10.2307/1592453

Käufer I, Weiss E (1980) Significance of bursa of Fabricius as target organ in infectious bursal disease of chickens. Infect Immun 27: 364-367

Kelly SM, Jess TJ, Price NC (2005) How to study proteins by circular dichroism. Biochim Biophys Acta - Proteins Proteomics 1751:119 139. https://doi.org/10.1016/J.BBAPAP.2005.06.005

Khan RSA, Sajid S, Habib M, Ali W, Salahudin SM, Sarfraz M (2017) History of Gumboro (infectious bursal disease) in Pakistan. Saudi Pharm J 25:453-459. https://doi.org/10.1016/j.jsps.2017.04.005

Kyte J, Doolittle RF (1982) A simple method for displaying the hydropathic character of a protein. J Mol Biol 157:105-132. https://doi. org/10.1016/0022-2836(82)90515-0

Lee C-C, Ko T-P, Chou C-C, Yoshimura M, Doong Ru S, Wang MY, Wang AHJ (2006) Crystal structure of infectious bursal disease virus VP2 subviral particle at $2.6 \AA$ resolution: implications in virion assembly and immunogenicity. J Struct Biol 155:74-86. https:// doi.org/10.1016/J.JSB.2006.02.014

Letzel T, Coulibaly F, Rey FA, Delmas B, Jagt E, Van Loon AA, Mundt E (2007) Molecular and structural bases for the antigenicity of VP2 of infectious bursal disease virus. J Virol 81:12827-12835. https://doi. org/10.1128/JVI.01501-07

Ley DH, Yamamoto R, Bickford AA (1983) The pathogenesis of infectious bursal disease: serologic, histopathologic, and clinical chemical observations. Avian Dis 27:1060. https://doi.org/10.2307/ 1590207

Mohamed MA, Elzanaty KES, Bakhit BM, Safwat MM (2014) Genetic characterization of infectious bursal disease viruses associated with Gumboro outbreaks in commercial broilers from Asyut province, Egypt. ISRN Vet Sci 2014:916412. https://doi.org/10.1155/2014/ 916412

Nielsen K, Yu WL, Lin M, Davis SAN, Elmgren C, Mackenzie R, Tanha J, Li S, Dubuc G, Brown EG, Keleta L, Pasick J (2007) Prototype single step lateral flow technology for detection of avian influenza virus and chicken antibody to avian influenza virus. J Immunoassay Immunochem 28:307-318. https://doi.org/10.1080/ 15321810701603443

Osajima T, Hoshino T (2016) Roles of the respective loops at complementarity determining region on the antigen-antibody recognition. 
Comput Biol Chem 64:368-383. https://doi.org/10.1016/J. COMPBIOLCHEM.2016.08.004

Owoade AA, Mulders MN, Kohnen J, Ammerlaan W, Muller CP (2004) High sequence diversity in infectious bursal disease virus serotype 1 in poultry and turkey suggests West-African origin of very virulent strains. Arch Virol 149:653-672. https://doi.org/10.1007/s00705003-0270-y

Pous J, Chevalier C, Ouldali M, Navaza J, Delmas B, Lepault J (2005) Structure of birnavirus-like particles determined by combined electron cryomicroscopy and X-ray crystallography. J Gen Virol 86: 2339-2346. https://doi.org/10.1099/vir.0.80942-0

Rahman M, Ismat F, McPherson MJJ, Baldwin SA (2007) Topologyinformed strategies for the overexpression and purification of membrane proteins. Mol Membr Biol 24:407-418. https://doi.org/10. 1080/09687860701243998

Rosa N, Ristic M, Seabrook SA, Lovell D, Lucent D, Newman J (2015) Meltdown: a tool to help in the interpretation of thermal melt curves acquired by differential scanning fluorimetry. J Biomol Screen 20: 898-905. https://doi.org/10.1177/1087057115584059

Sastre P, Pérez T, Costa S, Yang X, Raber A, Blome S, Goller KV, Gallardo C, Tapia I, Garcia J, Sanz A, Rueda P (2016) Development of a duplex lateral flow assay for simultaneous detection of antibodies against african and classical swine fever viruses. J Vet Diagn Investig 28:543-549. https://doi.org/10.1177/ 1040638716654942

Shah MA, Ullah R, De March M, Shah MS, Ismat F, Habib M, Iqbal M, Onesti S, Rahman M (2017) Overexpression and characterization of the $100 \mathrm{~K}$ protein of Fowl adenovirus- 4 as an antiviral target. Virus Res 238:218-225. https://doi.org/10.1016/j.virusres.2017.06.024

Singh NK, Dey S, Madhan Mohan C, Mohan Kataria J, Vakharia VN (2010) Evaluation of four enzyme linked immunosorbent assays for the detection of antibodies to infectious bursal disease in chickens. J Virol Methods 165:277-282. https://doi.org/10.1016/j.jviromet. 2010.02.008

Stoute ST, Jackwood DJ, Sommer-Wagner SE, Crossley BM, Woolcock PR, Charlton BR (2013) Pathogenicity associated with coinfection with very virulent infectious bursal disease and infectious bursal disease virus strains endemic in the United States. J Vet Diagnostic Investig 25:352-358. https://doi.org/10.1177/1040638713483538

Sun YL, Yen CH, Tu CF (2014) Visual detection of canine parvovirus based on loop-mediated isothermal amplification combined with enzyme-linked immunosorbent assay and with lateral flow dipstick. J Vet Med Sci 76:509-516. https://doi.org/10.1292/jvms.13-0448

Taghavian O, Spiegel H, Hauck R, Hafez HM, Fischer R, Schillberg S (2013) Protective oral vaccination against infectious bursal disease virus using the major viral antigenic protein VP2 produced in Pichia pastoris. PLoS One 8:e83210. https://doi.org/10.1371/journal.pone. 0083210

Ullah R, Shah MA, Tufail S, Ismat F, Imran M, Iqbal M, Mirza O, Rahman M (2016) Activity of the human rhinovirus 3C protease studied in various buffers, additives and detergents solutions for recombinant protein production. PLoS One 11:e0153436. https:// doi.org/10.1371/journal.pone. 0153436

Vakharia VN, He J, Ahamed B, Snyder DB (1994) Molecular basis of antigenic variation in infectious bursal disease virus. Virus Res 31: 265-273. https://doi.org/10.1016/0168-1702(94)90009-4

Vanden BTP, Gonze M, Morales D, Meulemans G (1996) Acute infectious bursal disease in poultry: immunological and molecular basis of antigenicity of a highly virulent strain. Avian Pathol 25:751-768. https://doi.org/10.1080/03079459608419179

Vanden BTP, Gonze M, Morales D, Meulemans G (2000) Acute infectious bursal disease in poultry: a review. Avian Pathol 29:175-194. https://doi.org/10.1080/03079450050045431

Wang W, Song Y, Liu L, Zhang Y, Wang T, Zhang W, Li K, Qi X, Gao Y, Gao L, Liu C, Zhang Y, Wang Y, Pan Q, He G, Wang X, Cui H (2019) Neutralizing-antibody-mediated protection of chickens against infectious bursal disease via one-time vaccination with inactivated recombinant Lactococcus lactis expressing a fusion protein constructed from the RCK protein of Salmonella enterica and VP2 of infectious bursal disease virus. Microb Cell Factories 18:21. https://doi.org/10.1186/s12934-019-1061-9

Wass JA (2002) Origin 7.0. Biotech Softw Internet Rep 3:130-133. https://doi.org/10.1089/152791602321105799

Whitmore L, Wallace BA (2004) DichroWeb, an online server for protein secondary structure analyses from circular dichroism spectroscopic data. Nucleic Acids Res 32:W668-W673. https://doi.org/10.1093/ nar/gkh371

Whitmore L, Wallace BA (2008) Protein secondary structure analyses from circular dichroism spectroscopy: methods and reference databases. Biopolymers 89:392-400. https://doi.org/10.1002/bip.20853

Zhang GP, Li MQ, Yang YY, Guo QJ, Li XW, Deng GR, Xiao JZ, Xing GX, Yang JF, Zhao D, Cai SJ, Min Zang W (2005) Development of a one-step strip test for the diagnosis of chicken infectious bursal disease. Avian Dis 49:177-181. https://doi.org/10.1637/7272090704R

Zorman RO, Barlic MD, Mitevski D, Lubke W, Mundt E (2003) Very virulent infectious bursal disease virus in southeastern Europe. Avian Dis 47:186-192. https://doi.org/10.1637/0005-2086(2003) 047[0186:VVIBDV]2.0.CO;2

Publisher's note Springer Nature remains neutral with regard to jurisdictional claims in published maps and institutional affiliations. 